\title{
How Form Errors Impact on 2D Precision Assembly with Clearance?
}

\author{
Pierre-Antoine Adragna ${ }^{1}$, Serge Samper ${ }^{2}$, and Hugues Favreliere ${ }^{2}$ \\ ${ }^{1}$ LASMIS, Université de Technologie de Troyes, 12 rue Marie Curie, 10010 Troyes, France \\ ${ }^{2}$ SYMME, Université de Savoie, BP 80439, 74944 Annecy le Vieux Cedex, France \\ adragna@utt.fr, \{serge.samper, hugues.favreliere\}@univ-savoie.fr
}

\begin{abstract}
Most models of assembling simulations consider that form errors are negligible, but how can this assumption be assessed? When clearances are high, form deviations can be neglected, but on the case of very precise mechanisms with small clearances, this assumption can lead to non-accurate models. This paper is the continuation of our previous works presented at IPAS 2008 dealing with the assembly of two parts regarding their form deviation. The proposed method considers the positioning of the pair of surface with a given external force to identify contact points. The parts relative positioning is expressed by a small displacement torsor that can be transferred to any referee and compared to the functional requirement. The objective of this paper is to identify the clearance domain of a mechanical linkage regarding the form deviation of parts. Several parameters are identified as influent such as the clearance value, the straightness of the form deviation and the localization of the ideal least squared associated shape.
\end{abstract}

Keywords: Form deviation, relative positioning, clearance domain, small displacement torsor, modal parameterization.

\section{Introduction}

Tolerancing of assembly can be solved using mathematical model of different levels of complexity. One very simple model presented by Graves [1] that only considers dimensional variations. More complex models are proposed to exploit the $3 \mathrm{D}$ tolerance zone, such as Chase [2] who proposes the use of the vector chain, or Davidson [3] who proposes the T-maps ${ }^{\circledR}$ model that can be compared to the small displacement torsor (SDT) proposed by Bourdet [4] and also used by Giordano [5] into the clearance domain model. These previous works consider that form deviation of parts can be neglected. Ameta [6] proposes to study the influence of form deviation based on the T-maps ${ }^{\circledR}$ model. Radouani [7] presents an experimental study of the positioning of parts regarding form deviations. Some mathematical methods are also presented to determine the relative positioning of parts regarding form deviations such as Neville [8], Stoll [9] and Morière [10]. Most of these approaches use optimization algorithm considering the minimization of a criterion based on distances or volumes between parts. 
Adragna [11] and [12] presents the static method that simply calculate the relative positioning of one shape on another considering their form deviations and a contact force, leading to the resolution of a mechanical static equilibrium. The main idea is to transfer the form deviation of the first shape to the second one in order to have a similar but simpler problem. Due to the form deviation, only few points of the shapes can be in contact. The identification of all possible set of contact points is obtained by the computation of a convex shape that filters and identifies the possible contact points and facets. The consideration of a positioning force allows identifying the contact facet. The positioning problem is then simplified to the identification of a geometric transformation in the small displacement domain leading the identified contact points of a shape on the contact points of the other one.

This paper is composed of three main parts. The first one presents the method for the relative positionning of parts regarding their form devition and a contact force direction. The second part presents the random generation of form deviation using the modal paramterization and the translation of the form deviation in two geometrical parameters. The third part presents the case of application that is a 2D linear linkage. A single study is firstly presented, and then simulations are drawn with differents values of form deviations. The paper ends by a conlusion.

\section{Proposed Method}

The assembly of one part on another is illustrated by the figures 1 and 2. Figure 1a shows two shapes with form deviation facing each other. Shapes are theoretically positioned and ideal shapes are associated by the least square criterion. Figure $1 \mathrm{~b}$ shows the first step of the method that transfers the upper shape form deviation to the lower one. The transferred deviation, also called distance surface, represents the point to point distances between the shapes. Shapes are in contact if two distances in a 2D problem are set to zero. Possible contact points and facets are identified thanks to the computation of the convex surface that filters the possible contact points. Figure $1 \mathrm{~b}$ shows two different force directions, force $a$ and force $b$, used to identify the contact facet and its contact points.
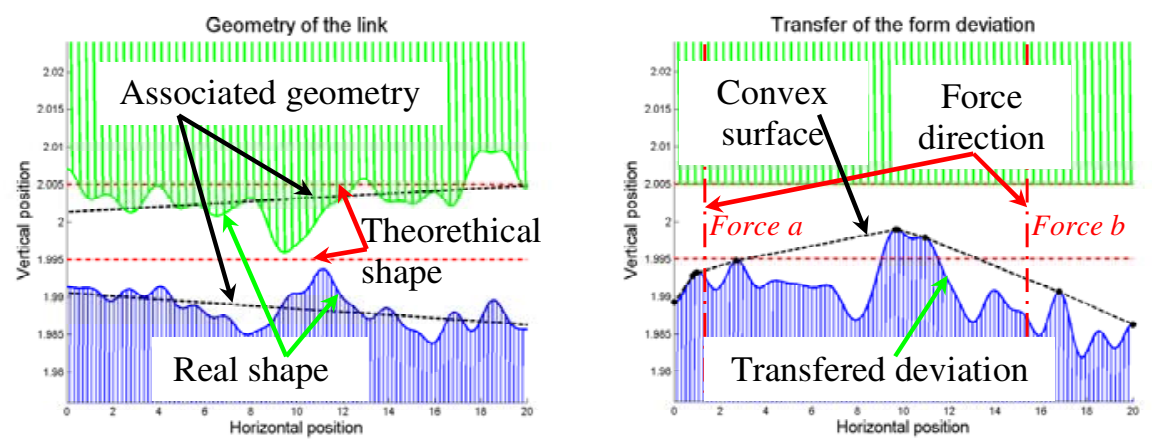

Fig. 1. a) two shapes with form deviation, b) transferring one shape form deviation on the other shape 
The following figure 2 shows two different positioning, figure $2 \mathrm{a}$ and $2 \mathrm{~b}$, depending on the force direction of force $a$ and force $b$ respectively.
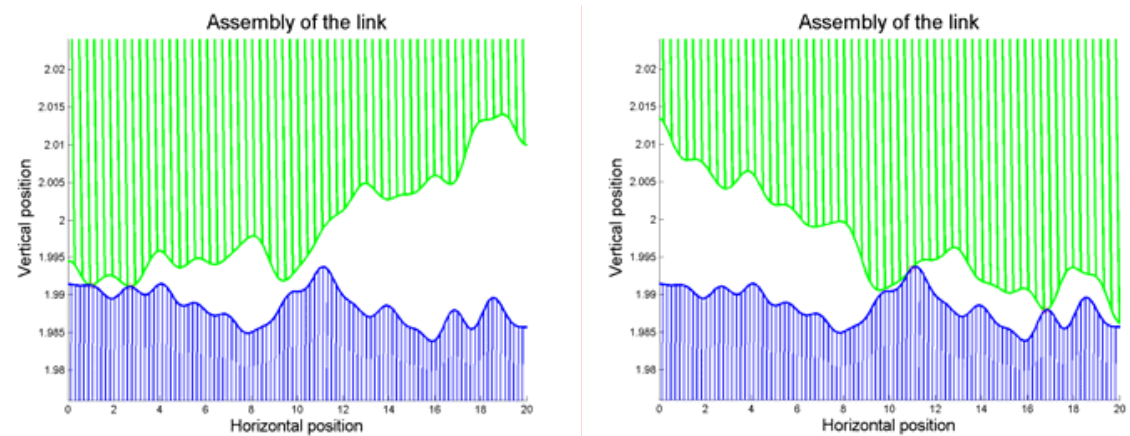

Fig. 2. Two contact configurations of shapes positioning, a) assembly for the direction of the given force $a, b$ ) assembly for the direction of the given force $b$

\section{Parameterization of Shapes}

This part presents two ways to parameter the form deviation. The first way is a description of the form deviation by elementary form deviation with a proposed method called the modal parameterization. The second way uses much simpler parameters that are geometrical zones.

\subsection{Modal Parameterization}

Samper [13-14] uses the modal analysis to generate a form deviation basis to characterize a measured form deviation. The main advantage of this method is that any type of geometry and form deviation can be characterized as a combination of elementary form deviation. The following figure 3 shows the height first modes of a 2D linear shape.

The analysis of a form deviation in the modal basis is a vectorial projection in a non orthogonal basis using the dual basis [15]. Hence, the result of the modal characterization of a measured form deviation $V$ on the $B$ modal basis is the modal signature $\Lambda$. This $\Lambda$ modal signature is composed of the $\lambda_{i}$ modal coefficients with metric meaning.

The recomposed shape with the rigid modes (translation and rotation) corresponds to the rigid shape and is equivalent to the Least Square associated ideal shape. The recomposed shape $R$ is obtained by the following relation:

$$
\mathrm{R}=\Lambda . \mathrm{B}
$$

\subsection{Random Shapes}

If a batch of produced part exists, then it is possible to draw simulations based on this family of form deviations. In other cases, assumptions can be made in order to define 

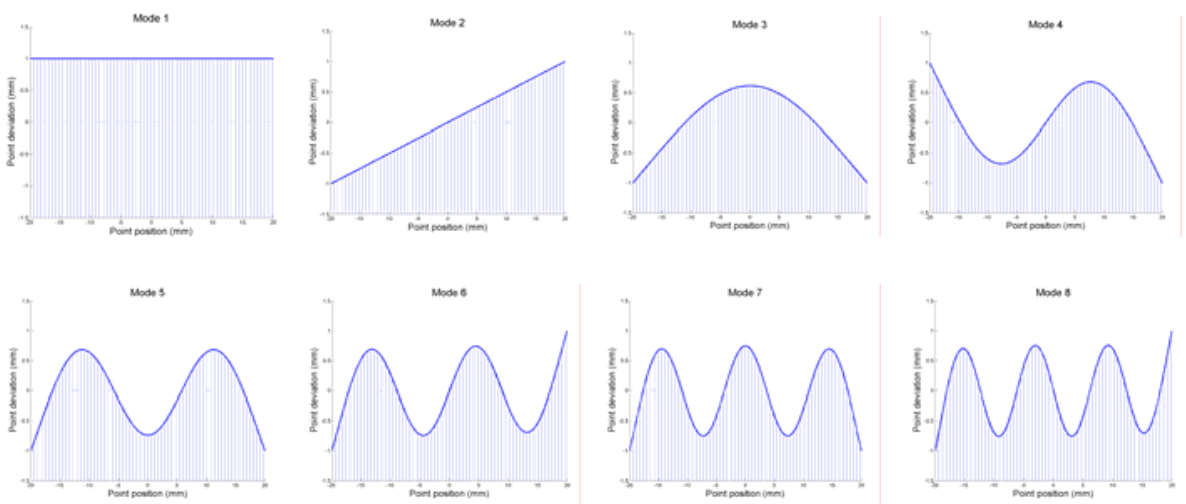

Fig. 3. First height modal vectors of a $2 \mathrm{D}$ profiles

the family shapes (batch of form deviations). The modal characterization of form errors can be used to create simulated shapes. A random draw of the modal coefficients creates a random shape which form complexity depends on the number of considered modes. Based on the observation of shapes analyses, amplitude of the modal coefficients is considered given by the following law:

$$
\mathrm{A}(\mathrm{i})=\mathrm{A}_{0} / \mathrm{i}
$$

Where $A_{0}$ is initial amplitude, $i$ is the order of the modal coefficient and $A(i)$ is the maximum amplitude of the $i^{\text {th }}$ modal coefficient. The following figure $4 \mathrm{a}$ shows the amplitude law of the coefficients and a random draw of a modal signature. This decreasing law can be changed in order to fit to a pilot production.

\subsection{Geometric Parameters}

The modal parameterization presented in this paper is only used to generate random shapes (lines in this case). Then two parameters are calculated, closer to geometrical specification:

- The strength of the shape that indicates the range of deviation,

- The localisation deviation of an ideal shape associated by the least square method.

The figure 4 represents the random drawing of the modal signature and the corresponding shape. On this shape is calculated the strength and the deviation of the associated geometry.

For this shape, the rectitude zone is $10.5 \mu \mathrm{m}$, the localisation deviation of the associated geometry is $3.8 \mu \mathrm{m}$ and the localisation deviation of the entire shape (including the form deviation) is $8.0 \mu \mathrm{m}$.

In order to identify the influence of parameters, shapes are randomly generated with modal signatures, then strength and localisations are measured. It is then possible to modify the modal signature thanks to an algorithm in order to set a given value to the shape rectitude and/or to the localisation of the associated geometry. 

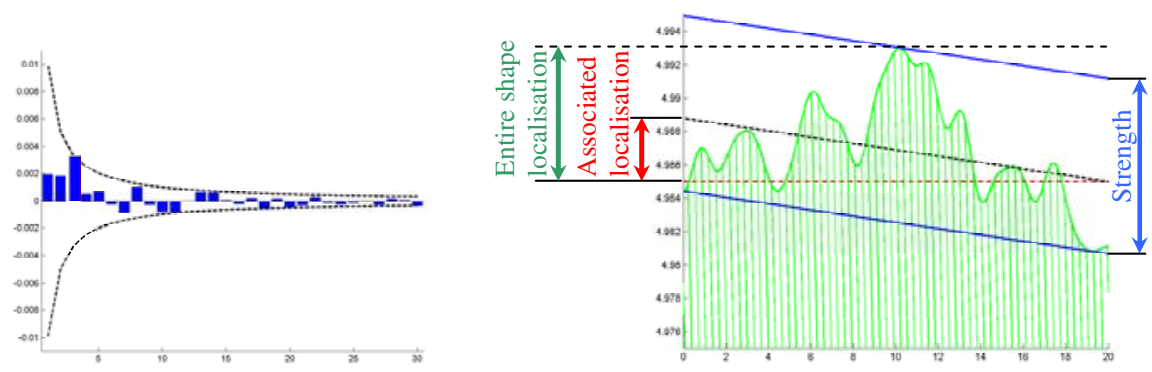

Fig. 4. a) randomly drawn modal signature, b) recomposed shape, the least square associated geometry and the rectitude zone

\section{Clearance Domain with Form Deviation}

This part presents our proposed method to find the clearance domain of a mechanical linkage regarding their form deviations based on the method detailed in the first part. A study case illustrates the method on a single application, and then simulations are drawn to compare our approach to the least square assembling model.

\subsection{D Linear Linkage}

To illustrate this paper, the study case is a 2D linear linkage, composed of two 2D linear contacts, upper and lower contacts. For this example, the geometrical clearance of the linkage is set to $20 \mu \mathrm{m}$ and form deviation are randomly drawn then measured.

Based on our proposed methods, possible contact facets and points are identified for each contact area, on the upper and lower.

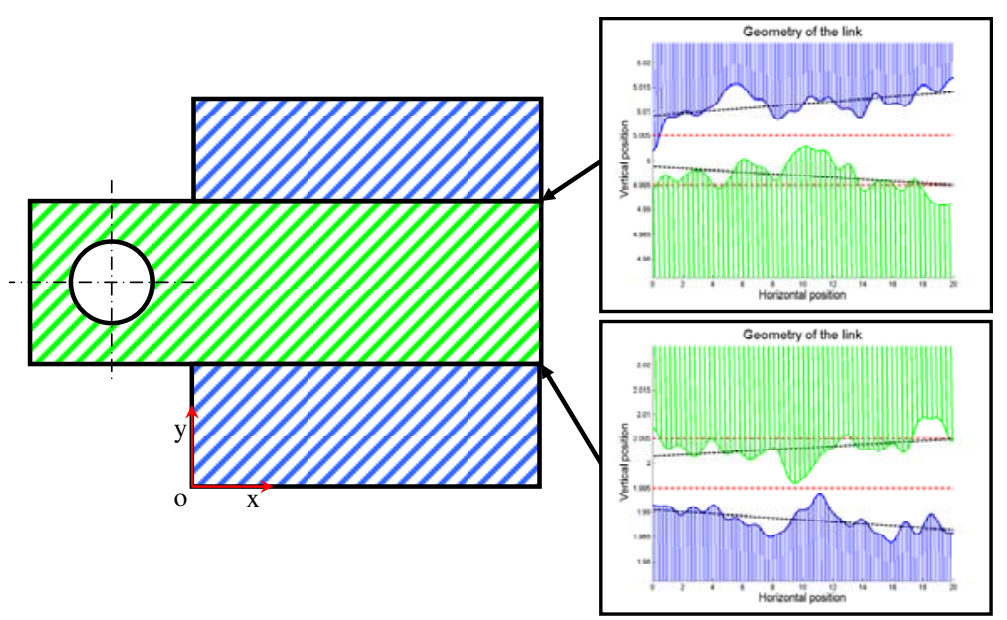

Fig. 5. Tthe mechanical linkage and its upper and lower contact zones 
Table 1. Values characterizing the shapes of the linkage

\begin{tabular}{|c|c|c|c|}
\cline { 2 - 4 } \multicolumn{1}{c|}{} & Strength $(\mu \mathrm{m})$ & Localisation $^{*}(\mu \mathrm{m})$ & $\begin{array}{c}\text { Real localisation } \\
(\mu \mathrm{m})\end{array}$ \\
\hline Shape 1 & 11.0 & 4.8 & 11.8 \\
\hline Shape 2 & 10.5 & 3.8 & 8.0 \\
\hline Shape 3 & 12.4 & 3.5 & 9.1 \\
\hline Shape 4 & 9.3 & 4.2 & 11.2 \\
\hline
\end{tabular}

deviation of the localisation of the least square associated geometry

An added difficulty is the fact that the linkage can not be assembled due interpenetration of parts. Hence, for each relative positioning of the inner part on the outer one, interpenetration is checked. The following figure 6 illustrates a positioning with interpenetration of parts; this relative positioning is no considered.
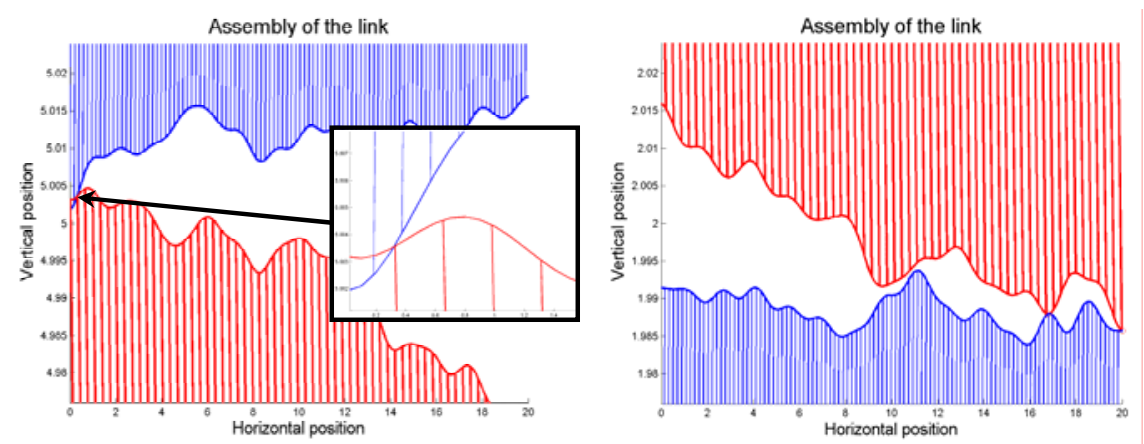

Fig. 6. Non-possible position of the linkage due to interpenetration on the upper contact, a) upper contact zone with interpenetration, b) lower contact zone with contact
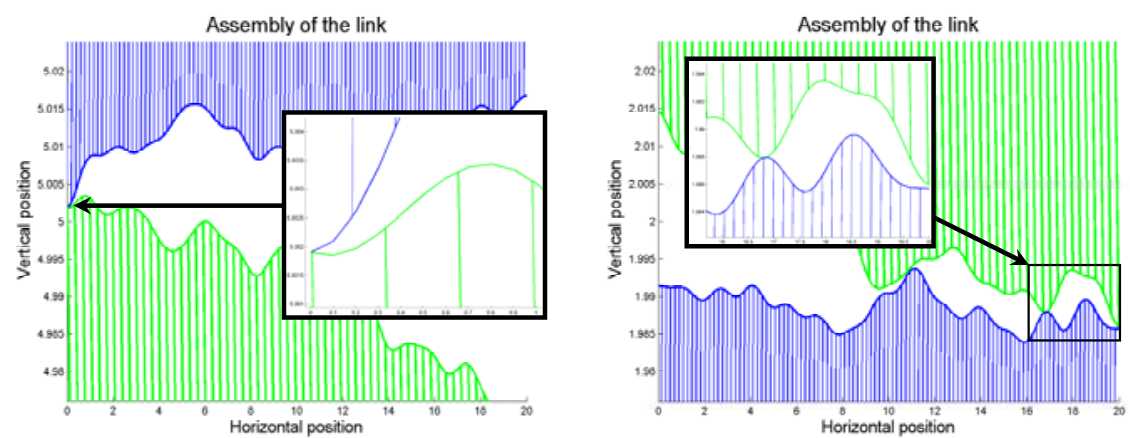

Fig. 7. One extreme rotation in the linkage, a) upper contact zone with contact, b) lower contact zone with contact 
Another difficulty is to find the two relative positioning given by the extreme rotations. An iterative approach is chosen to find both extreme rotations in the linkage. The following figure 7 shows one extreme rotation where contact points are not located at the extremity of the shapes.

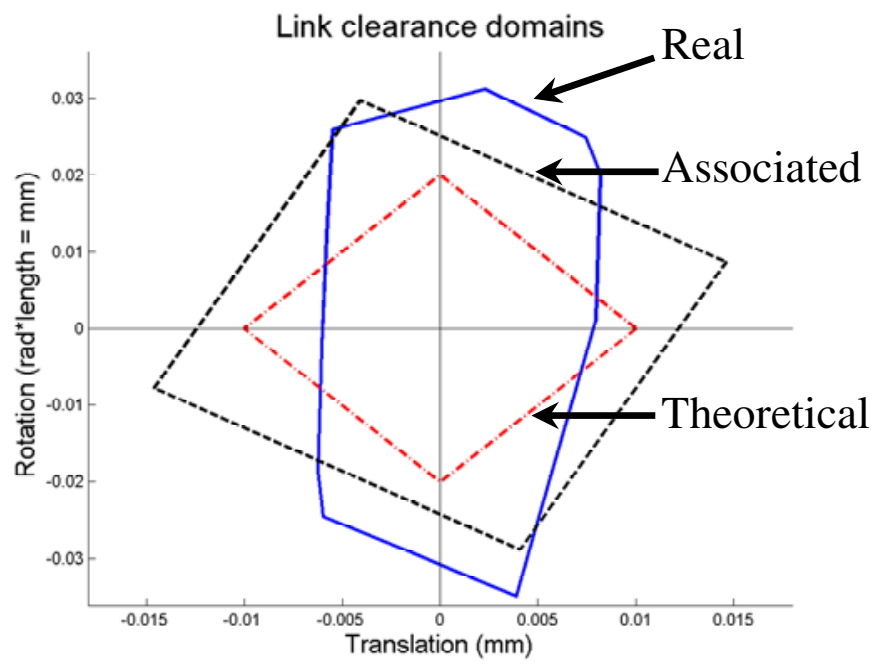

Fig. 8. Linkage clearance domains for theoretical, associated and real model

Finally, the clearance domain of the mechanical linkage is identified and showedon the following figure 8. In this graph, small displacement torsors components define the axis. Limits are always convex (small displacement assumption). Three domains are obtained:

- The red one corresponds to the theoretical clearance domain given by the geometrical characteristics of the linkage and its gap,

- The black domain corresponds to the clearance domain obtained with the ideal least square associated shapes, which is the commonly used model,

- The blue domain corresponds to the real clearance domain regarding to the form deviation of shapes. This domain is defined by the convex domain of the parts relative positioning in the small displacement domain where interpenetration positions are not used but extreme rotations are added.

Table 2. Characteristics of the theoretical, associated and real clearance domains

\begin{tabular}{|c|c|c|c|}
\cline { 2 - 4 } \multicolumn{1}{c|}{} & Area $\left(\mu m^{2}\right)$ & Rotation range $(\mu \mathrm{m})$ & $\begin{array}{c}\text { Translation range } \\
(\mu \mathrm{m})\end{array}$ \\
\hline Theoretical & 400 & 40 & 20 \\
\hline Associated & $925(=231 \% *)$ & $58.6(=147 \% *)$ & $29.3(=147 \% *)$ \\
\hline Real & $755(=189 \% *)$ & $66(=165 \% *)$ & $14.5(=73 \% *)$ \\
\hline
\end{tabular}

compared to the theoretical clearance domain 
To compare these different clearance domains, it is chosen to measure the domains area, translation range (horizontal length) and rotation range (vertical length). The following table 2 shows the different domains measures where it can be observed that, for this particular case, the theoretical domain area is more or less half of the associated and real domains areas. Another remark is that the associated domain is almost homothetic compared to the theoretical one, but the real domain shows that although the rotation range is a bit larger than the translation range of the associated domain, the translation range is half of the translation range of the associated domain.

\subsection{Simulations}

This part presents simulations in order to evaluate the influence of the strengthness and associated localization zones. The clearance value of the mechanical link is set to $6 \mu \mathrm{m}$. Strengthness and associated localization of shapes are independantly set from 0 to $12 \mu \mathrm{m}$ by step of $2 \mu \mathrm{m}$. For each configuration, 1000 assemblies are drawn, and their associated and real clearance domains are measured. Hence, the following results are made by $7 * 7 * 1000$ simulations.

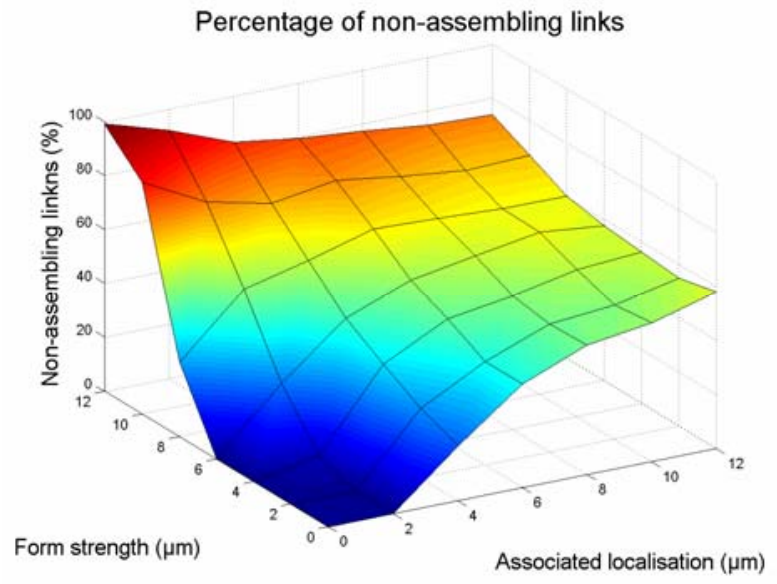

Fig. 9. Evolution of the rate of non-assembling linkages depending on the forms strengths and associated localisation for the $6 \mu \mathrm{m}$ linkage clearance value

The first result showed in the figure 9 is the rate of non-assembling linkage given the deviation zones of shapes find with the real model considering the form deviation. It can be observed that almost all linkage can be assembled when the associated zone is null and the shape strength is lower or equal to the linkage clearance.

The next figures concern the dimensions (area, translation and rotation range) of the associated and real clearance domains. Plotted surfaces represent the mean dimensions of the clearance domains of the drawn assemlies.

It can be observed on the following figure 10 that the domains dimensions are identical to the theoretical domains when no associated deviation are considered, this is due to the model that does not consider form deviation. Another remark is that 
dimensions of the domains grow as deviation zones grow, there is no compensation due to the strengthness it can be observed on our model as showed in the following figure 11 .

The figure $11 \mathrm{c}$ shows that when the associated deviation zone equals or is upper to the linkage clearance, the strength almost has no effect on the rotation range of the clearance domain. However, as the strength increases the real clearance domains characteristics deacrease.
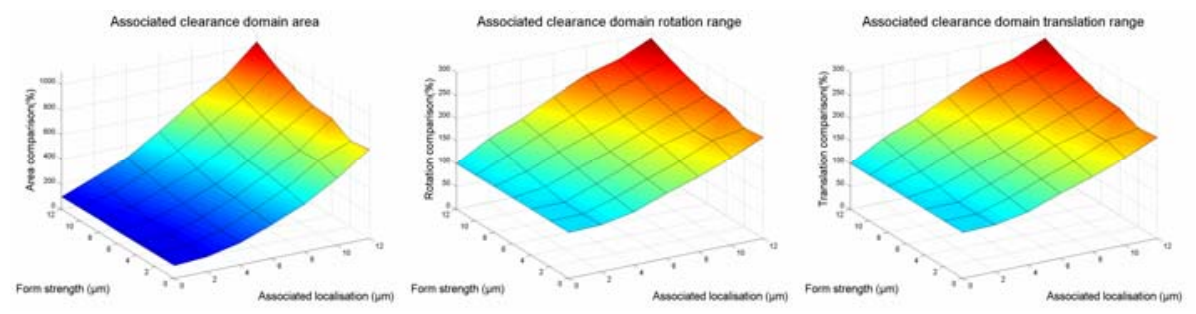

Fig. 10. Evolutions of the associated clearance domains, a) domain area, b) domain rotation range, c) domain translation range
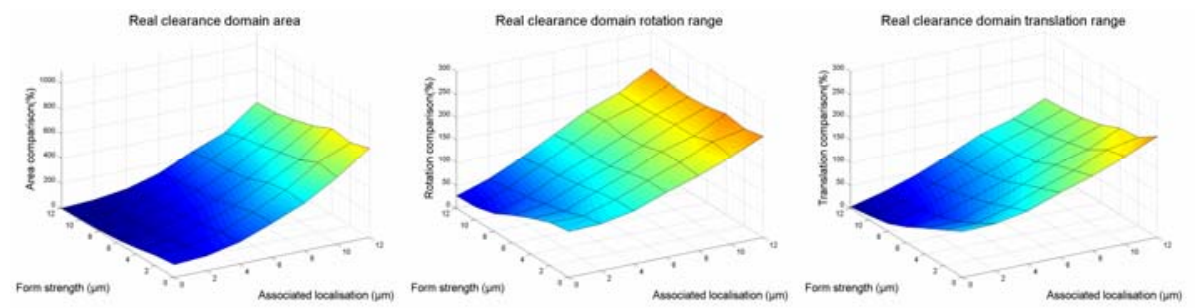

Fig. 11. Evolutions of the real clearance domains, a) domain area, b) domain rotation range, c) domain translation range

\section{Conclusion}

This paper continues our previous works dealing with form deviations in assemblies and shows that form deviations of parts not only impact their relative positioning but also impact the assembly clearance domain of the mechanical linkage.

It can be conclude that the rigid assembly model usually considered for tolerancing is not correct concerning the assembly prediction with form deviation, even in a small deviation zone. Hence the solution proposed by [6] and others, the strength deviation is considered as an additional rigid deviation zone, can appear to be a correction of this problem.

Then, works have to be continued to evaluate more precisely the separated and combined influence of each form deviation on the assembly functional requirement. The aim will be to find an expression or a criterion for the acceptance of the shape deviation that guarantees the functional requirement. 


\section{References}

1. Graves, S.: Tolerance Analysis Formula Tailored to Your Organization. Journal of Quality Technology 33(3) (2001)

2. Chase, K.W.: Tolerance Analysis of a 2D and 3D assemblies, ADCATS Report, No 94-4 (1999)

3. Davidson, J.K., Shah, J.J.: Geometric Tolerances: A New Application for Line Geometry and Screws. ImechE Journal of Mechanical Engineering Science 216, Part C, 95-104 (2002)

4. Bourdet, P., Mathieu, L., Lartigue, C., Ballu, A.: The Concept of Small Displacement Torsor in Metrology. In: Proceedings of the International Euroconference, Advanced Mathematical Tools in Metrology, Oxford (1995)

5. Giordano, M., Kataya, B., Pairel, E.: Tolerance Analysis and Synthesis by Means of Clearance and Deviation Spaces. In: Proceedings of the 7th CIRP International Seminar on Computer Aided Tolerancing, pp. 345-354 (2001)

6. Ameta, G., Davidson, J.K., Shah, J.: Influence of Form Frequency Distribution for a 1D Clearance Wich is Generated from Tolerance-Maps. In: Proceedings of the 10th CIRP Conference on Computer Aided Tolerancing, Specification and Verification for Assemblies, Erlangen Germany (2007)

7. Radouani, M., Anselmetti, B.: Contribution à la Validation du Modèle des Chaînes de Cotes - Etudes Expérimentale du Comportement de la Liaison Plan sur Plan. In: Congrès International Conception et Production Intégrées, Meknès Moroco, October 22-24 (2003)

8. Neville, K.S.L., Grace, Y.: The modeling and analysis of butting assembly in the presence of workpiece surface roughness part dimensional error. International Journal of Advanced Manufacturing Technologies 31, 528-538 (2006)

9. Stoll, T., Wittman, S., Meerkamm, H.: Tolerance Analysis with detailed Part Modeling including Shape Deviations. In: Proceedings of the 11th CIRP Conference on Computer Aided Tolerancing, Geometric Variations within Product Life-Cycle Management, Annecy France (2009)

10. Morière, S., Mailhé, J., Linares, J.-M., Sprauel, J.-M.: Assembly method comparison including form defect. In: Proceedings of the 11th CIRP Conference on Computer Aided Tolerancing, Geometric Variations within Product Life-Cycle Management, Annecy France (2009)

11. Adragna, P.-A., Samper, S., Favrelière, H., Pillet, M.: Analyse d'un assemblage avec prise en compte des défauts de forme. In: Congrès International Conception et Production Intégrées, Rabat Morocco (2007)

12. Adragna, P.-A., Favrelière, H., Samper, S., Pillet, M.: Statistical assemblies with form errors - a 2D example. In: Proceedings of the 4th International Precision Assembly Seminar (IPAS 2008), Micro-Assemblies Technologies and Applications, Chamonix France, pp. 23-33 (2008)

13. Formosa, F., Samper, S., Perpoli, I.: Modal expression of form defects. In: Models for Computer Aided Tolerancing in Design and Manufacturing, pp. 13-22. Springer, Heidelberg (2007)

14. Adragna, P.-A., Samper, S., Pillet, M.: Analysis of Shape Deviations of Measured Geometries with a Modal Basis. Journal of Machine Engineering: Manufacturing Accuracy Increasing Problems - Optimization 6(1), 95-102 (2006)

15. Favrelière, H., Samper, S., Adragna, P.-A., Giordano, M.: 3D statistical analysis and representation of form error by a modal approach. In: Proceedings of the 10th CIRP Conference on Computer Aided Tolerancing, Specification and Verification for Assemblies, Erlangen Germany (2007) 